\title{
Políticas para microempresas en las gobernaciones venezolanas: el caso del estado Falcón
}

\author{
López Carrasco, Carlos E. * \\ Ochoa Henriquez, Haydée C.**
}

\section{Resumen}

Las políticas subnacionales de apoyo a las microempresas han sido poco estudiadas en Venezuela. Este trabajo explora tres programas de apoyo a las microempresas puestos en práctica en el Estado Falcón en los años noventa. El punto de partida es la existencia de una estrecha relación entre economia y administración pública, aunque a través de un proceso complejo derivado de la autonomía que esta última adquiere. Los principales resultados revelan: a) Que formalmente los programas buscan constituir una politica social productiva que contemplaba estrategias de comercialización y hasta de exportación, pero en la práctica no consideraron esos propósitos, b) Una clara orientación de la politica en los tres programas hacia el nuevo rol que la reestructuración neoliberal le asigna al Estado, c) Influencia de organismos financieros intemacionales y de estrategias de financiamiento foráneas, d) Tendencia a la aplicación del modelo gerencial tecnocrático, en el marco de resistencias burocrático-populistas. Se concluye que: 1) Existe una ten* dencia al apoyo a las microempresas bajo los criterios propios del Estado neoliberal, favorecida por influencias y experiencias intemacionales, pero con limitaciones que le imponen las aspíraciones de los gobemadores a mantenimiento y ascenso en el poder, 2) No se vislumbraron altemativas a favor del desarrollo socio-económico integral y de profundización de la democracia como ha pretendido trasmitir el discurso de la descentralización y, 3) Se evidencia lo poco factible que resultó en la década de los noventa la repolitización de los espacios públicos subnacionales, hecho que parece tomar nuevos rumbos a partir de la importancia que en la nueva constitución se asigna a las cooperativas en la descentralización.

Palabras clave: Microempresas, gobernaciones, políticas públicas subnacionales, Estado Falcón, Descentralización, Venezuela.

Fecibido: 00-10-20. Aceptado: 00-12-14

* Politólogo, Magíster en Gerencia Pública. Profesor de Post-grado en Políticas Públicas. Investigador del Centro de Estudios de la Empresa de La Universidad del Zulia (LUZ)

E-Mail: lopez.carlos@terra.com.ve

* Dra. en Estudios del Desarrollo. Investigadora del Centro de Estudios de la Empresa de LUZ en el área de Administración Pública. Coordinadora del Doctorado en Ciencias Sociales en LUZ Acreditada en el Programa de Promoción al Investigador (PPI) del CONICIT.

E-Mail: haydeeochoa iamnet.com 


\title{
Microenterprise Policy in State Governments in Venezuela: Falcon State Case
}

\begin{abstract}
Sub-national support policies for microenterprise received little study in Venezuela. This paper explores support programs for microenterprise that were initiated in Falcon State in the decade of the nineties. The starting point is an analysis of the close relationship between economy and public administration, because of a complex process related to the autonomy of the latter. The principle results of the study reveal that: a) formally the programs focus on constituting a social productive policy and contemplate strategies for commercialization and even exportation, but in reality, these purposes are not considered, b) there is a clear orientation of policies in three programs that promote the new role of neo-liberal structuring on the part of the state, $c$ ) there is influence from intemational financing organisms and foreign financial strategies, d) there is a tendency to apply technocratic management models in the framework of populist bureaucratic resistance. The conclusions are that: 1) there is a tendency to support microenterprise under the criteria of a neo-liberal state, which is favored by international experiences and influences, but with limitations which are imposed by the state governors to maintain and increase their power, 2) There are no alternatives on the horizon in favor of integrated economic and social development, or the deepening of democracy which decentralizing discourse has tried to transmit, and 3 ) there is evidence of little effectiveness in the decade of the nineties in terms of policy change in sub-national political arenas, a situation which may take on more importance due to the emphasis in the new constitution of cooperatives in decentralization.
\end{abstract}

Key words: Microenterprises, state government, sub-national public policy, Falcon State, decentralization, Venezuela.

\section{Introducción}

Con la reestructuración económica iniciada en Venezuela a fines de los ochenta, ha tenido lugar un proceso de reforma del Estado el cual condujo a cambios significativos tanto en la política como en la administración estatal.

En este contexto, las gobernaciones son receptoras de las competencias que el nivel nacional le traslada con el proceso de descentralización político-territorial, el cual constituye uno de los ejes fundamentales de la reforma del Estado. Tales instituciones pasan a asumir funciones económicas y sociales que hasta entonces no tenían, una de ellas ha sido la de diseñar y poner en práctica políticas de apoyo a las microempresas, con lo cual se insertan en las nuevas políticas sociales que la reestructuración económica ha generado.

Las microempresas son unidades de producción que al igual que las gobernaciones, toman importancia con el proceso de reestructuración económica, al formar parte de la política social compensatoria del Estado, aunque formalmente se presentan como una estrategia de inversión social o productiva que intenta ir más allá de los típicos programas compensatorios y focalistas donde la gratui- 
dad exige al Estado una permanente intervención. Producto de la política para microempresas, se crea el Programa de Apoyo a la Economía Popular ${ }^{1}$, que viene a constituir junto con el Fondo de Cooperación y Financiamiento de Empresas Asociativas (FONCOFIN) ${ }^{2}$, las instancias de la administración pública central responsables del apoyo a las microempresas en el país.

El apoyo del Estado a las microempresas ha recibido la atención de numerosos investigadores en el pais (Barrantes, 1997; Freitez, 1991; Iranzo, 1990) entre otros; sin embargo el papel de las gobernaciones en este apoyo no ha sido estudiado, lo que a nuestro juicio constituye un vacío en el conocimiento de este fenómeno social, si consideramos que con el proceso de descentralización político territorial las gobernaciones han asumido numerosas tareas que tienen que ver tanto con la dimensión económica como con la social.

El presente estudio tiene el propósito de analizar la política formulada por parte de la Gobernación del Estado Falcón para apoyar a las microempresas en la referida entidad federal. A raíz del proceso de descentralización político-territorial la Gobernación del Estado Falcón ha puesto en práctica tres programas de apoyo a las microempresas: 1) EI Programa de Microempresas del Estado Falcón (MICROEMFAL), 2) El Programa de In dustria Familiar (PIF) y, 3) el Programa de las Cajas Rurales, los cuales tienen lugar en organismos diferentes de la Goberna- ción y tiempos distintos; sin embargo, tal como señala Subirats (1989) no por ello dejan de conformar una política pública, permitiendo, en concordancia con Oszlak y O'Donnell (1976), que de dicha intervensión gubernamental se pueda inferir una cierta direccionalidad, una determinada orientación normativa, que preferiblemente afectará el futuro curso del proceso social.

Partimos del supuesto de que existe una estrecha relación entre la economía y la política pública (Ochoa, 1997), además, tal como señala Mény y Thoenig (1991: 101) "...cada vez, más numerosos estudiosos de políticas públicas han puesto en evidencia la importancia de las instituciones y del Estado como organizaciones a través de las cuales los agentes públicos (elegidos o administrativos) persiguen objetivos que no son exclusivamente respuesta a las demandas sociales, así como configuraciones de organizaciones $y$ de acciones que estructuran, modelan e influyen tanto los procesos económicos como las clases o grupos de interés".

De ahí que consideremos que las políticas de apoyo a la microempresa tiene una orientación funcional a la reestructuración neoliberal de la economía. En su implementación se intenta imponer el paradigma gerencial-tecnocrático que se corresponde con los requerimientos económicos de la nueva política, el cual debe enfrentarse al modelo burocrático-populista hasta ahora dominante y vinculado a la economia para el consumo interno y a

1 Sustituido en 1995 por el Programa de Fomento a la Economia Solidaria.

2 Organismo del gobierno central adscrito al Ministerio de Familia; en la actualidad ninguno de estas instancias forman parte del aparato público. 
los requerimientos de la democracia acordada por los partidos signatarios del Pacto Punto $\mathrm{Fijo}^{3}$.

\section{Gobernaciones y microempresas en los noventa}

Con la aprobación en Venezuela de la Ley de Delimitación, Descentralización y Transferencia de Competencias del Poder Público (LODDT) en 1989, la cual se sustentó en el traslado de competencias del nivel central al estadal ${ }^{4}$, las gobernaciones o gobiernos provinciales, que desde su origen fueron fundamentalmente organizaciones político-militares para el control territorial, pasan a ser organizaciones de gran complejidad, inmersas en una nueva dinámica socio-política; en la cual se plantea que los gobiernos subnacionales asuman un papel más relevante en la gestión económico-social del Estado.

"Aunado a la complejidad derivada de nuevas funciones, tenemos que como parte de la reforma del Estado, los Gobernadores dejaron de ser designados ejecutivamente por el Presidente de la República y son elegidos por votación directa, con lo cual se modifican las relaciones de poder de las gobernaciones con el nivel central, así como con la sociedad civil, lo que añade nuevos elementos de com- plejidad a estas instancias del aparato público" (Ochoa, 1999:71)

La magnitud de la importancia que adquieren las gobernaciones en el plano económico se deja ver en el hecho que pasan a administrar en condiciones exclusivas la infraestructura de apoyo a la economía: puertos, vialidad, aeropuertos y puentes. En el plano social se insertan en condiciones compartidas con otros niveles del aparato estatal en la administración de las políticas compensatorias y focalistas diseñadas por el Estado para disminuir los problemas sociales que la reestructuración económica ha incrementado, pero también la mayoría de las Gobernaciones toman iniciativas en el diseño e implementación de políticas sociales bajo su única dirección, en lo cual inciden por una parte los compromisos que asumen los gobernadores en los procesos electorales, asi como a las aspiraciones de continuidad y ascenso en el poder una vez que resultan electos al cargo de gobernador. Por otra parte, con los cambios las gobernaciones son objeto de mayor demanda de satisfacción de necesidades sociales, lo que tiene lugar en el marco de un discurso oficial que le asigna al proceso de descentralización político territorial propósitos de profundización de la democracia.

Al igual que se producen cambios en las gobernaciones a raíz de la rees-

3 Pacto acordado en Venezuela en el año 1958 que "involucra a AD, COPEI y URD, FEDACAMARAS, la CTV, la Igiesia y las Fuerzas Armadas...los partidos...controlan al Estado y movilizan...y regulan el funcionamiento de la 'sociedad civil', es decir el resto de la sociedad y sus organizaciones: sindictos, gremios, asoclaciones diversas etc." (Urbaneja; 1984:229)

4 A diferencia de la mayoria de los paises de América Latina, en los cuales la descentralización político-territorial descansó en el nivel municipal. 
tructuración económica, tienen lugar significativas modificaciones a la política de apoyo a las microempresas. Según Barrantes (1999:39), el financiamiento del Estado venezolano a las iniciativas económicas de los sectores populares se remonta a 1959, con los inicios del Pacto de Punto Fijo, al crearse la Comisión Nacional de Crédito al Artesanado y la Pequeña Industria, la cual fue reestructurada en 1961 como Comisión Nacional de Financiamiento a la Pequeña y Mediana Industria. En 1974, debido a su descapitalización, ésta fue clausurada y sustituida por la Corporación Industrial (CORPOINDUSTRIA). Agrega el autor (1999:40), que el fomento para actividades económicas a sectores de escasos recursos, tuvo a lo largo de todo el período democrático puntofijista readjetivaciones tales como: economía de base, social, asociativa, popular y solidaria; sin embargo según Barrantes, estas nociones, siendo cooperantes en la práctica del buen gobierno y la buena sociedad, son asumidas como excluyentes en la antitética práctica gubernamental de borrón y cuenta nueva.

En el contexto de incremento de los altos índices de desempleo y pobreza, en las últimas dos décadas, se han propiciado en el país distintas alternativas de sobrevivencia y búsqueda de ingresos por parte de amplios sectores de la población, incrementándose el llamado sector informal de la economia o trabajo por cuenta propia, circunstancia frente a la cual desde mediados de los ochenta en el Gobierno de Jaime Lusinchi, el Estado venezolano suscribió con el PNUD el Proyecto "Superación de la Pobreza", del cual surgen lineamientos programáticos para la economía popular (Barrantes; 1997:147), esto evidencia la influencia de los organismos internacionales en estas políticas. Estos lineamientos se constituyen formalmente en política pública en 1987 con la creación de FONCOFIN, poco antes de ponerse en práctica las medidas de reestructuración económica.

Con el proceso de ajuste y reestructuración económica iniciado en 1989 , que intenta modificar la estrategia de crecimiento económico de sustitución de importaciones basada en el Estado de bienestar, instrumentada a lo largo de todo el mencionado período del "Pacto de Punto Fijo"; por una economia de mercado, se reorienta la política social con el Plan Nacional de Enfrentamiento a la Pobreza, enmarcado en el VIII Plan de la Nación, en cuyo contexto se creó el Programa de Apoyo a la Economia Popular, junto a otros cinco programas.

De manera que la política de apoyo a las microempresas elaborada por el Estado en 1987, a través de FONCOFIN, es fortalecida con la creación del mencionado Programa de Apoyo a la Economía Popular en 1989, el cual tenia una concepción, como dice Barrantes (1999:42), sustentada sobre la base de un microempresario individual, altamente productivo y hasta tecnologizado con capacidad exportadora, contrario con la concepción asociativista inspirada en la economía social del Gobierno de Jaime Lusinchi (1984-1989), del cual surge FONCOFIN.

Bajo esta circunstancia, las microempresas como unidades productivas de la Economia Popular pasan a formar parte de la agenda de prioridades nacionales y de la agenda de gobiernos subnacionales, con la creación de organismos y 
programas estadales para el sector, sustentada en un discurso que plantea una política social distinta de la tradicional forma de gestión universalista, subsidiaria, burocrática, desarticulada, y costosa.

Es asi como a inicio de los noventa los gobiernos estadales o provinciales diseñaron y ejecutaron programas de apoyo a las microempresas que contemplan teóricamente el respaldo en forma integral en materia de asistencia técnica, financiera y capacitación, destinados al fomento económico de unidades productivas de grupos sociales en situación de pobreza. Los motivos para el desarrollo de este tipo de programas en las Gobernaciones venezolanas están relacionados, según Avila y Freitez (1992: 16-17) con la necesidad de ofrecer respuestas frente a los problemas del desempleo de la fuerza de trabajo y a los bajos ingresos de los grupos familiares de extrema pobreza. A nuestro juicio, en el contexto de los cambios que se produjeron en las gobernaciones.

Las gobernaciones al ser financieramente dependientes del situado constitucional, ingresos que en su gran parte son destinados a gasto corriente, han debido recurrir para la inversión económica, a fórmulas de organización intergubernamental, como el Fondo Intergubernamental para la Descentralización (FIDES), el Fondo de Inversión Social (FONVIS) o la Ley de Asignaciones Especiales, los cuales no contemplan los programas de microempresas, por lo que deben "negociar" sus recursos con los organismos centrales tales como el referido FONCOFIN.

En este contexto, las acciones que en los años 1989-1990 se proponen ejecutar las gobernaciones para el apoyo a microempresas con programas independientes del Ministerio de Familia, se vinculan a éste en la negociación de recursos financieros. (Avila y Freitez 1992: 2)

Por su parte, a nivel nacional el primer programa gubernamental de Apoyo a la Economía Popular desarrollado por FONCOFIN, y puesto en práctica en el contexto descentralizador de inicio de los noventa, permitió combinar esfuerzos del nivel central, de las gobernaciones, alcaldias y organizaciones no gubernamentales. Para Avila y Freitez (1992:19-20) este proceso "en un corto periodo de ejecución ha hecho posible apoyar a nivel del país más de diez mil unidades microempresariales desarrolladas por sectores de bajos ingresos; tales resultados no hubieran sido posibles con las pautas del esquema de ejecución centralizada".

En la ejecución de este programa se unieron dos tipos de iniciativas; por una parte, la de los gobiernos estadales o provinciales y locales, vinculadas a la necesidad de dar respuestas a los requerimientos de fomento económico de sus electores. La autonomía de los nuevos gobiernos estadales y locales ha hecho posible destinar recursos financieros $y$ humanos con tales propósitos. Por otra parte, la ejecución descentralizada del programa que se impulsa desde FONCOFIN, permitió completar las iniciativas locales con recursos, asistencia y orientaciones para la planificación, ejecución y seguimiento.

La confluencia de tales iniciativas permitió el planteamiento de gestión conjunta en la instrumentación de estos programas de apoyo a la microempresa; $\sin$ embargo, la necesidad de legitimidad de los gobiernos recién electos para esa épo- 
ca, plantea a ellos, el requerimiento de generar políticas propias para crear empleos y estímulo a las actividades económicas de sectores de bajos ingresos. Por el deterioro que vienen presentando las condiciones generales de vida de estos sectores sociales, los gobiernos estadales y locales están obligados a buscar respuestas adecuadas, y las políticas de apoyo a las pequeñas iniciativas económicas locales son una opción que está demostrando su adecuación a estas circunstancias.

Desde una perspectiva más amplia de esta situación Saraví (1998) ha planteado dos posibles alternativas, a partir del proceso de reconversión de la economía: una de encumbramiento y otra de declive.

La primera, parte de la experiencia de los distritos industriales exitosos europeos, como por ejemplo los de la Tercera Italia, el Baden-Württember en Alemania y algunas regiones del Sur de España, zonas en las cuales el agrupamiento de pequeñas empresas ha favorecido la creación de un capital social que genera redes de colaboración y complementariedad en los ámbitos subnacionales o locales, como elementos determinantes de la flexibilidad y competitividad. La concentración socioterritorial no hace referencia simplemente a la aglomeración productiva de firmas especializadas en un mismo sector, sino también a la presencia de una atmósfera sociocultural e institucional que favorece a la generación de redes productivas de cooperación.

\section{Para Piore (citado por Saravi,} 1998) las áreas prioritarias de política pública que se desprenden de los análisis sobre estos distritos industriales euro- peos son, por un lado la provisión de servicios reales, que se refiere a la provisión directa o indirecta de los gobiernos subnacionales y de las organizaciones intermedias de una amplia gama de servicios que van desde la investigación y desarrollo, la capacitación laboral y la educación, hasta el marketing, las consultorías, la compras de insumos, e incluso los servicios de salud y otros beneficios sociales, hasta, el establecimiento de reglas y estándares de comportamiento consensados, que sirven como guías o directrices para el desarrollo de la economía local, cuya principal función sería la de reducir la explotación entre los miembros de la comunidad, que podrían atentar contra las relaciones de cooperación, por ejemplo, leyes locales encaminadas a evitar la competencia salarial, procedimientos para distribuir la demanda, para asegurar el pleno empleo y aprovechar los recursos de la comunidad.

La alternativa de declive, según Saraví tiene lugar fundamentalmente a partir de políticas macroeconómícas de apertura de mercados que contribuyen a acentuar el proceso de exclusión de las micro y pequeñas empresas en los esfuerzos por insertarse a formas organizativas más dinámicas, planteándose como una de las mayores preocupaciones para ese sector, la de multiplicar las estrategias de sobrevivencia, lo cual conlleva a que la estrategia de la competitividad se haya traducido en una precarización de las condiciones de vida y trabajo, bajo el estímulo del escenario "sálvese quien pueda".

Para el mencionado autor (1998:259), siguiendo a Enrique de la Garza, esto es "flexibilidad primitiva", "...basada en la discrecionalidad patro- 
nai y la desprotección legal, lo cual dista mucho de significar que se trata de relaciones de cooperación consensadas". Plantea que la segunda alternativa está basada en principios neoliberales de apertura y desregulación.

De todo lo anteriormente planteado es importante destacar, que para el autor las micro y pequeñas empresas son potenciales agentes de desarrollo económi$\mathrm{co}$, bajo el requisito sine cua non de la construcción de canales participativos y democráticos para el diálogo y la obtención del consenso a nivel local, de lo que se desprende que del conjunto de políticas industriales locales y regionales deberían ser el resultado de la cooperación entre gobiernos, asociaciones de productores y organizaciones de trabajadores que participan conjuntamente y en un clima de buenas relaciones laborales dentro del amplio espectro de las instituciones locales (Saraví, 1998: 260).

\section{EI Programa MICROEMFAL}

A inicio de la década de los noventa el Ejecutivo del Estado Falcón complementó las políticas nacionales contra el desempleo, con la creación de dos Fondos de Financiamiento a las pequeñas y medianas empresas: El Fondo Estadal de Crédito Agrícola (FONECRA) y el Fondo de Financiamiento de Artesanos y a la Pequeña y Mediana Industria (FONDAPEMI) ${ }^{5}$ y además casi paralelamente com- pletó recursos financieros para la creación y puesta en marcha del Programa de Apoyo a la Microempresa del Estado Falcón (MICROEMFAL). Como antecedente a todo ello podemos mencionar la Coordinación de la Pequeña y Mediana Industria y la Artesanía, perteneciente al Comité de Coordinación y Planificación (COPLAN FALCÓN), creado a principios de 1990.

Estas modalidades de intervención subnacional para el desarrollo económico y social comienzan a ofrecerse desde 1989 , año en el que se diseñó una oferta al pueblo de Falcón, en el marco de la histórica campaña electoral, donde por primera vez se elegían en Venezuela, en forma directa: Gobernador del Estado, Alcaldes y Concejales. Ese momento fue el escenario, según FONDAPEMI (s.t.), en el cual se asumió el compromiso de atender los requerimientos de los sectores productivos a fin de poner en marcha programas de acción que reactivaran la deprimida economía del Estado.

\subsection{MICROEMFAL y la política económica neoliberal}

Este programa nace a partir de un convenio suscrito entre FONCOFIN y CORPOFALCON en Septiembre de 1990, surge de la estrategia de descentralización de FONCOFIN a inicios de los noventa para impulsar el Programa de Apoyo a la Economía Popular; el cual, se

5 A partir de la elección de los gobernadores han proliferado organizaciones dependientes de la gobernación, con personalidad jurídica propia, dedicadas al manejo de recursos financieros para desarrollar programas especiales dirigidos a atacar algunos problemas en diversos segmentos del entomo económico de la entidad; en el Estado Falcón se han creado tres institutos 
presenta como una política social renovada. Para el Equipo Técnico Operativo de MICROEMFAL (Mestre; et al, 1991) la creación de este programa se justifica por el comportamiento reciente de la economia venezolana, que ha afectado de manera significativa a los grupos familiares, en especial los sectores de escasos recursos; por lo tanto, se dirigió a la ejecución de proyectos que brindaran a las pequeñas unidades de producción y servicio el acceso al financiamiento para incrementar la producción a fin de mejorar el nivel de ingreso y la posibilidad de generar empleo.

Iranzo et al (1990:25) plantean que la noción de lo informal presenta confusiones y ambigüedades que como categoría empírica, agrupa demasiadas situaciones diferentes, como para ser útil en la formulación de políticas. Ante ello, se introduce la noción de Economia Popular como categoría básica que conceptualiza el apoyo del gobierno al sector informal en el VIII Plan de la Nación, denominado "Gran Viraje", a nuestro juicio correspondiente con la nueva política económica neoliberal.

La principal diferencia que destaca a primera vista, entre MICROEMFAL y los otros programas de la Gobernación para enfrentar el problema del empleo y el desarrollo económico local, adscritos a FONDAPEMI y FONECRA, es su población objetivo. Las unidades económicas formalmente atendidas por MICROEMFAL según Mestre et al (1991: 5) fueron sólo personas o grupos organizados de escasos recursos que habitaban en barrios urbanos o zonas rurales, con experiencia laboral y manejo del oficio principal de la actividad para la cual solicita apoyo. La población objetivo de FONDAPEMI Y FONECRA en sus inicios, fue la pequeña y mediana empresa, particularmente empresarios establecidos en la región, cuyas características económicas están distantes de los microempresarios.

El programa se formuló para atender tres áreas: 1)Capacitación en herramientas básicas para ser competitivo en el mercado, 2)Asistencia Técnica en administración, producción y comercialización la cual estaba prevista realizarse a través de visitas periódicas y atención a consultas y 3) Financiamiento, en el que nos detendremos, por ser el área que ocupó mayor atención.

Se definieron dos tipos de crédito: 1) Pequeños Crédito con un monto máximo de Bs. 150.000 , una tasa anual del $18 \%$ y un período de gracia 3 meses y 2 ) Créditos de Desarrollo con dos categorías, una cuyo monto oscilaba entre Bs. 150.000 y 300.000 bolivares y otra con montos entre Bs. 300.000 y 500.000 .

Las condiciones de financiamiento establecidas fueron las siguientes: 1) La actividad económica a desarrollar por el beneficiario debe garantizar un margen de ganancia minima que permita la devolución del crédito, 2) Los rubros de financiamiento definidos fueron: Capital de trabajo, maquinarias y equipos, 3) La activi-

autónomos desde fines de los ochenta para prestar apoyo a las microempresa: La Corporación de Desarrollo del Estado Falcón (CORPOFALCON), creada en 1987, FONDAPEMI, creado en 1990 y FONECRA, creado en 1990. 
dad debía ser realizada en el Estado Falcón, en una primera fase comprendía los Municipios Miranda, Colina, Bolivar, los Taques y Punta Cardón, en una segunda fase estaba previsto cubrir todo el estado, 4) Los solicitantes debian laborar directamente en la actividad económica para la cual solicitan apoyo 5) Sólo se otorgaría un crédito por familia, 6) Los solicitantes no deberían amortizar ningún crédito de programas similares para la unidad, 7) $\mathrm{EI}$ valor de los activos fijos y constituidos de las microempresas no deberían pasar de Bs. 500.000 Bs. 8) Se tendría preferencia por las actividades económicas que estuvieran dirigidas a satisfacer las necesidades básicas de sus dueños, 9) El solicitante debía presentar garantías, tales como reservas de dominio de maquinarias y equipos; fianzas quirogralarias o prendarias y aval (MICROEMFAL; 1993).

La estrategia de financiamiento utilizada por el MICROEMFAL fue la creación de un fondo de garantía, que según Barrantes (1996: 27) son depósitos realizados en un ente bancario a los efectos de avalar, subsidiaria y transitoriamente, el otorgamiento de préstamos a clientes sin garantías reales. Las empresas que resultasen beneficiarias del programa MICROEMFAL, obtendrían la liquidación de sus créditos previa apertura de cuentas de ahorro en las instituciones financieras seleccionadas.

Con estas condiciones estamos en presencia de criterios que toman distancia de las orientaciones de política pública en la etapa de la economía por sustitución de importaciones, estos criterios apuntan a garantizar la recuperación del préstamos a diferencia de las tradicionales prácticas financieras del Estado populista. Asumi- mos se trata de un programa que pone en evidencia que nos encontramos ante un programa que se ubica en la alternativa que hemos denominado partir de los aportes hechos por Saravi de "declive".

\subsection{El papel del PNUD}

Según Vilas (2000:46), "no es ningún secreto para nadie que las recomendaciones de política pública formuladas por el FMI, el Banco Mundial, el BID y organismos similares inciden decisivamente en la matriz de poder de la sociedad, promoviendo a algunos actores, discriminando contra otros, y en definitiva, interviniendo en la dinámica política y social en nombre de una racionalidad técnica o de los macroeconomics fundamentals".

El programa MICROEMFAL no ha escapado de esta influencia de los organismos internacionales. Por una parte está la concepción del programa, el cual como dijimos en páginas anteriores nace de la asesoria del PNUD al Proyecto Superación de la Pobreza, donde surgen los lineamientos programáticos para la Economía Popular, en el que se inserta el programa MICROEMFAL, financiado en un $50 \%$ por FONCOFIN inicialmente. La inversión del programa originaimente fue de 30 millones de bolivares, de los cuales 15 financió la mencionada institución y el resto la Gobernación del Estado Falcón a través de CORPOFALCON. A partir de 1992 el programa fue financiado exclusivamente por la Gobernación, hasta 1993 año en el cual fue cerrado.

Por otra parte encontramos que la estrategia financiera programada y descrita en el punto anterior, es la recomendada por el PNUD. Esta influencia se da a 
través de la asesoria que la Fundación para el Desarrollo de la Región Centro Occidental (FUDECO) prestó a MICROEMFAL; la primera institución coordinó la subcomisión de Economia Popular del Plan Nacional de Enfrentamiento a la Pobreza donde participaron además FONCOFIN y Consultores del PNUD y del Instituto Latinoamericano de Investigación Social (ILDIS). Dicha metodología fue ensayada en el Estado Lara por FUDECO y por el Fondo de Financiamiento para Pequeños Artesanos y Pequeños Talleres de Producción, también en el Estado Lara.

Como podemos observar a pesar de que la Gobernación de Falcón no convino directamente con los organismos internacionales para la programación y ejecución del programa MICROEMFAL, hubo uno influencia de los criterios del Programa de las Naciones Unidas para el Desarrollo (PNUD). Este organismo, principal fuente de financiación del Sistema de Cooperación Técnica de las Naciones Unidas, según Pérez Salgado (1997:251), en el curso de la década pasada, conjuntamente con otros organismos internacionales como el Banco Interamericano de Desarrolio (BID) y el Banco Mundial (BM), han hecho importantes contribuciones en torno a la gobernabilidad, promoviendo el enfoque de la Gerencia Pública y específicamente cuestiones como: la descentralización de Estado, la reforma de la administración de justicia, cambios institucionales, la reforma de los partidos políticos y la lucha contra la corrupción. A nuestro juicio, son procesos que están avanzando vinculados a la necesidad de reformar el aparato público en el contexto de la globalización.

\subsection{Los obstáculos burocráticos-populistas}

En la práctica el programa MlCROEMFAL se administró con las viejas prácticas del modelo burocrático-populista adoptadas en Venezuela desde los inicios de la democracia, cuyo principal instrumento de legitimación es el gasto público. Dicho modelo se caracteriza además por una alta intermediación de los partidos políticos, los cuales a partir del Pacto de Punto Fijo debían impulsar el modelo de sustitución de importaciones y el bienestar, necesarios para la legitimación; sin embargo el rol de los partidos previsto originalmente, terminó desvirtuándose, en función de intereses de éstos, a los cuales se subordinaron los requerimientos del experimento democrático iniciado al comienzo de los sesenta.

La aplicación de criterios populistas en el programa MICROEMFAL se expresa en la gestión de los recursos financieros. Por un lado, durante su corta vida, el programa destinó a costos operativos, parte de los recursos previstos para los créditos; por otro lado, tal como señala un funcionario de la gobernación, los créditos fueron otorgados con mediación partidista (Sánchez, 2000), todo lo cual condujo finalmente a la descapitalización del programa. Finalmente, se liquidaron 151 créditos, distribuidos en diez municipios del Estado Falcón, por un monto total de $33.451 .388,00$ bolivares, de los cuales se lograron recuperar desde sus inicios en 1991 hasta diciembre de 1993, la cantidad de $9.768 .780,00$ bolívares, es decir que los créditos en su mayoría iban a fondo perdido, Todo lo anterior cual revela la 
existencia de un estilo de gestión burocrático-populista.

Esta situación, consideramos que da cuenta de obstáculos a una estrategia social diseñada para dar respuestas a las exigencias de un nuevo entorno económico, que requiere sustentación en el modelo de gestión gerencial-tecnocrático.

La descapitalización del programa codujo a que FONCOFIN no aportara más recursos; hecho que llevó, según su Equipo Técnico, a solicitar al Ejecutivo Regional un aporte de 40 millones bolivares a partir de lo cual se autofinanciaria el programa. Esta situación no se llegó a concretar, lo que condujo a la desaparición del MICROEMFAL.

La gobernación no volvió a poner en práctica un programa para microempresas, hasta la vigencia del Programa de Industria Familiar (PIF), donde se observa una preocupación por no repetir la gestión bajo los parámetros anteriores.

\section{El Programa de Industria Familiar (PIF)}

Luego del fracaso del programa MlCROEMFAL la Gobernación del Estado Falcón quedó sin una estrategia definida para atender al sector microempresarial. Es con otro gobierno iniciado en 1995, socialcristiano al igual que su antecesor, cuando en el marco del III Plan de Desarrollo para el Estado Falcón (1996-1998), se propone como parte del Desarrollo
Económico, el establecimiento de una política integral en materia industrial, que contempla dos operaciones que sientan las bases conceptuales para la reformulación de la política sobre microempresas; por una parte se plantea "propiciar la inserción de la producción industrial regional en el proceso de globalización internacional", por otra parte, "la promoción y el fortalecimiento de la microempresa de producción y de la artesania, para lo cual se establece el diseño de un programa de Asistencia Técnica y Financiera para la Microempresa de Producción" (COPLAN, 1996:39-40).

\subsection{EL PIF y la política económica neoliberal}

En el contexto expuesto se crea, según Decreto No. 13 del 30 de Enero de 1997 (Curiel, 1997), el Programa de Industria Familiar (PIF), el cual constituye una reformulación a la política de apoyo a las microempresas por parte de la Gobernación del Estado Falcón.

Los actores involucrados así como la motivación de esta reformulación se dejan ver en entrevista realizada a la Asesora de Desarrollo Económico de la Gobernación, quien planteó lo siguiente: "...una de las primeras líneas de acción que dio el Gobernador a su equipo económico fue montar un "banco para la mujer" que tuviera un funcionamiento sencillo, y a esa tarea nos dedicamos Carios Curiel ${ }^{6}$ y yo" (Castro;2000). Agregó Castro, que para

6 El ling. Carios Curiel tue Presidente de FONDAPEMI y FONECRA y también formó parte, conjuntamente con el ing. Rodolfo Sánchez, funcionario de FONDAPEMI, de la Comisión Presidencial para el Estudio y Formulación de Políticas para el Fomento Microempresarial y Desarrollo de Sistemas de Microcréditos que el 14 de Abril de 1998 designó el Presidente Rafael Caldera. 
esto se asesoraron con la ONG: Centro de Formación Popular Renaciendo Juntos (CEPOREJUN), la ONG más importante en el Estado Falcón para el desarro. llo de iniciativas microempresariales.

Surge asi el PIF con recursos de la gobernación, sin recursos de FONCOFIN, a pesar de haberlos solicitado. EIPIF se adscribe formalmente a FONDAPEMI y FONECRA, aunque en la práctica lo asume solamente el primer organismo.

Las áreas del PIF son: Asistencia técnica pre y post-financiamiento y financiamiento, al igual que el programa MlCROEMFAL se contemplaba la realización de talleres de mercadeo y competitividad en el caso del PIF como parte de la asistencia técnica. A diferencia de MICROEMFAL, el PIF no contemplaba la comercialización de los productos como parte de la asistencia técnica. Tal como hicimos con el programa MICROEMFAL, nos detendremos en el área financiamiento.

Las condiciones de financiamiento fueron las siguientes: 1) Los rubros a financiar son exclusivamente para la adquisición de materia prima y herramientas menores, 2) Las actividades productivas a financiar son: a) Artesania: Articulos de cuero, orfebreria, madera y piedra, arcilla y gres, cerámica, alpargatas, zapateria, hamacas, muñequería; b) Producción: Corte y confección, carpintería, fabrica de bloques, repostería, fabrica de hielo, comida rápida, c) Servicios: Peluqueria, talleres mecánicos, reparación de electrodomésticos, tapicería, plomería y electricidad, serigrafía y d) Comercio: Abastecimiento Popular. 3) El monto a otorgar por cada crédito es hasta de un máximo de ciento cincuenta mil bolivares en el caso de las microempresas urba- nas, y hasta un máximo de un millón de bolivares en el caso de los créditos agrícolas. 4) Las condiciones de pago son: Seis meses de plazo en la primera etapa y doce meses para la segunda etapa, pagaderos en cuotas mensuales ajustables trimestralmente, 5) La garantía viene dada por la responsabilidad colectiva del grupo solidario y giros firmados por cada uno de los clientes y sus cónyuges, 6) Para optar al crédito se requiere: a) Estar realizando la actividad para la cual solicita el financiamiento, b) Pertenecer a un grupo solidario, c) Asistir a la charla informativa y al taller plan de negocios y d) Asumir compromisos de seguimiento permanentes y 7) El financiamiento se otorga a actividades económicas establecidas, a fin de lograr su consolidación definitiva y garantizar de esta forma la recuperación del crédito en el tiempo previsto.

Una cuestión medular de las condiciones de financiamiento del PIF la constituye la creación de los llamados Grupos Solidarios, como un mecanismo que permite garantizarle a la institución el retorno del crédito otorgado. Estos grupos se plantean como un conglomerado de cinco personas dedicadas a actividades económicas iguales o distintas, de manera ordinaria y a pequeñas escalas, que de forma espontánea deciden agruparse para constituir un aval recíproco aunado a un criterio de asociatividad que considere: conocimiento mutuo trato, amistad, vecindad, responsabilidad y solidaridad.

A diferencia de la etapa inicial de la política con el MICROEMFAL, cuyo discurso se centró claramente en la solución de problemas sociales, el Plan de Desarrollo Regional de 1996 se planta explícitamente que las microempresas sean 
funcionales a las dinámicas de la globalización económica, insertadas en una estrategia general de industrialización. Sin embargo, para FONDAPEMI, los objetivos del PIF giran en torno a la solución de problemas de empleo de grupos económicos vulnerables y desarrollo del trabajo familiar en el ámbito comunitario; esto pone de manifiesto, como señala Socorro (1995:18), las dificultades para la conceptualización de las microempresas y evidencia, como dice Barrantes (1996: 27), la ubicación de los objetivos de los programas microempresariales tanto en la esfera económica como en la social.

La posición de FONDAPEMI no es obstáculo para que los funcionarios de esta institución opinen que las microempresas deberían ir hacia la constitución de empresas bajo la figura de Compañía Anónima, de manera que puedan optar por créditos más grandes, cuestión que apunta a insertar a las microempresas en la dinámica de acumulación,

Por lo que, de lo planteado hasta ahora se revela, como dicen varios autores (Bossier, 1989; De Mattos, 1989), que la descentralización territorial resulta un proceso correlacional con la reestructuración neoliberal de la economia, y su pertinencia para la productividad como fin primordial y lógico del proceso.

En efecto, esta participación de las gobernaciones venezolanas en el desarrollo de microempresas se explica porque "a pesar de las tendencia a minimizar el papel del Estado en la producción directa de bienes y servicios y de que las propuestas más radicales le asignan un rol pasivo en la economia, la práctica ha demostrado que al menos en los paises subdesarrollados el Estado tiene una participación acti- va, en el proceso de reestructuración económica" (Ochoa, 1999: 73), y para Medellín Torres (citado por Ochoa, 1999:78) porque "la nueva corporeidad del Estado debe estar definida por la misión, razón y práctica empresarial de las relaciones entre la sociedad y el Estado".

Por lo tanto, la puesta en práctica del PIF implica por un lado, una importante vía para relegitimar la acción gubernamental subnacional si tomamos en cuenta que son los sectores populares menos favorecidos, los que están bajo esas circunstancias; pero también a medida que la racionalidad económica convierte a la financiación de una actividad medio a un fin, esta forma de intervención gubernamental se mercantiliza, haciendo que dicha legitimidad se subordine a las necesidades de acumulación.

En ese sentido, son pertinentes las críticas que De Mattos (1989:125) hace al proceso de descentralización cuando señala lo siguiente: "la dinámica socioeconómica capitalista está afectada por condicionamientos estructurales que no pueden ser removidos por la vía de la simple reorganización territorial del poder; no hay fundamento teórico ni empírico, que respalde el supuesto de que el descenso hacia lo local en una sociedad capitalista, conduzca a situaciones propensas al predominio de los intereses populares".

\subsection{La influencia de estrategias internacionales en el PIF}

El Programa de Industria Familiar, en su cuestión medular, es decir en la estrategia de grupos solidarios, tiene una gran influencia de orientaciones puestas en práctica a nivel internacional desde los 
años ochenta, a raíz del proceso de reestructuración económica; surge de los también llamados prestamos mancomunados; cuyos antecedentes se remontan hacia los años setenta, cuando Mahummad Yunus, profesor de economía de la Universidad de Chittangong en Bangladesh, le prestó el equivalente de treinta dólares a una serie de artesanos suprimidos en la pobreza extrema quienes dependian de los usureros para adquirir material (Yunus; 1998) ${ }^{7}$.

En América Latina muchos programas de créditos para microempresas adoptaron desde principios de los ochenta el mecanismo de los grupos solidarios, como plan de garantía para superar las restricciones institucionales y los elevados costos de transacción del crédito para empresas sumamente pequeñas (McKean, 1988:153).

Según Otero (1988: 127) las instituciones que utilizan los grupos solidarios en sus actividades adaptan dicho mecanismo a su filosofía y le añaden componentes para su efectividad. Agrega el autor, que entre las innovaciones que presenta este enfoque se encuentra: la movilización del ahorro, el suministro de servicios adicionales (como atención a la salud, ayuda legal, alfabetización y programas de mejoras de viviendas) y el énfasis en llegar a los subsectores de microempresarios, como los microvendedores, $o$ a determinada línea de microproductores ${ }^{8}$.

Sin embargo, no se puede concluir únicamente a partir de la implantación de esta metodología que se trata de una acción del Estado despolitizada, en tal sentido es conveniente destacar en palabras del mismo Yunus: “. .somos partidarios de reducir la intervención del Estado. Apoyamos la economía de mercado y la creación de empresas", agrega el autor: "Grameen no es adepto al laissez-faire. Creemos en la intervención social, pero sin que el Estado se involucre en la industria o los servicios. Su intervención deberia limitarse a un conjunto de medidas que alienten a la empresa a comprometerse en el ámbito social".

Lo anterior evidencia la orientación del Banco Grameen, a pesar de que Yunus intenta demostrar lo contrario cuando señala sobre la afirmación anterior: "Esto podría situarnos a la derecha. Sin embar-

7 Producto de esta experiencia se creó el Banco Grameen que para mediados de los noventa tenía una cartera superior a los 2.000 millones de dólares y el sistema de créditos mancomuna. dos se ha extendido a más de 52 países, y constituye una muestra de que a personas pobres se le puede prestar dinero sin los criterios que se plantean a partir del crédito bancario tradicional. (Yunus, 1998).

8 También según la referida autora, varía la importancia que dan las instituciones a la participación activa de los miembros del grupo en el programa. En ciertos programas, los grupos solidarios existentes tienen a su cargo la promoción de los mismos y ayudan a la formación de los nuevos grupos. Asimismo, contribuyen al desarrollo del material de capacitación adecuado y de planes de estudio para su utilización junto con el otorgamiento del crédito. En los programas más avanzados, como en Cartagena en Colombia, y Santo Domingo en República Dominicana, se considera a los grupos solidarios como un paso intermedio hacia un nivel más amplio de organización entre los beneficiarios de los programas, quienes, como resultado de ello forman cooperativas (Otero, 1998:127). 
go, el Grameen defiende los objetivos sociales: eliminar la pobreza, dar a todos educación, cobertura médica y empleo, contribuir a la igualdad de los sexos permitiendo que las mujeres se hagan cargo de si mismas, y asegurar el bienestar de las personas mayores. Grameen sueña con un mundo sin pobreza y sin limosna de los subsidios, y cuestiona el marco ins. titucional existente y las empresas fundadas sólo en la búsqueda de la ganancia" (Yunus, 1998:225). Se trata a nuestro juicio de estrategias sociales necesarias para la legitimación y la gobernabilidad del Estado, especialmente en la etapa neoliberal.

Evidencia la afirmación anterior, el hecho que el organismo que pone en práctica la metodología de los grupos solidarios a partir del PIF, que es FONDAPEMI, no tiene como filosofía principal el conformar una gama de servicios alternos como los que señala Otero; este organismo es un instituto financiero, que aunque de carácter público, debe, a partir del paradigma de la búsqueda de eficiencia lograr suficiente margen de recuperación de los créditos para garantizar "significativos resultados económicos" y administrativos que hagan viable su gestión.

A su vez, la principal orientación que se tiene con los grupos solidarios no es la conformación de cooperativas, a pesar de que recientemente se plantea impulsar la "asociatividad" para desarrollar algunos sectores económicos del Estado Falcón (Castro, 1999).

En el piano concreto la influencia de la Estrategia de los Grupos Solidarios en el PIF se da a través de la asesoría de CEPOREJUN, una ONG que ha demostrado gran audacia para obtener fondos de instituciones públicas nacionales tales como FONCOFIN y Petróleos de Venezuela S. A. (PDVSA) y de organismos internacionales tales como: Embajadas de Canadá y Finlandia, Fundación Interamericana de Desarrollo, Broederlijk Denle y la Comunidad Económica Europea entre otros. CEPOREJUN utiliza la estrategia mencionada para suministrar financiamiento a microempresarios de la región, a su vez su referente en la materia lo constituye la Fundación Carvajal de Colombia.

\subsection{Tendencia tecnocrática del PIF}

De la primera etapa de puesta en practica de este programa en el año 1997, según un estudio realizado por Sánchez López (1998) en el Municipio Miranda (el cual abarcó el $87.8 \%$ de la cobertura total) sobre su impacto socio-económico, se encontraron entre otros los siguientes resultados: Experiencia laboral en la actividad desarrollada; administración realizada por los mismos beneficiarios del crédito, el $78 \%$ destinó el crédito a la actividad económica para la cual solicitaron el financiamiento, y el $22 \%$ a la realización de actividades no contempladas en el programa, los beneficiarios no han buscado integrarse para formar organizaciones, se recuperó el $77 \%$ de capital invertido en el Municipio Miranda, la recuperación se explica en parte, por la gestión para la cobranza del Fondo y a la posibilidad de obtener un segundo financiamiento.

Este cuadro evidencia profundas diferencias respecto a las prácticas populistas en las cuales el financiamiento del Estado iba a fondo perdido y con grandes desviaciones de sus propuestas iniciales. 
En el año de 1998, se lograron atender con el PIF 583 microempresas distribuidas en 14 municipios del Estado Falcón. Lo cual significo una inversión de 116.800 .000 bolivares., lo que a su vez representó el $65 \%$ de los montos ejecutados por los programas de FONDAPEMI, que según información proveniente de este mismo organismo ello significó la generación de 1166 empleos (FODAPEMI; 1998, 1999, 2000).

A pesar de esta tendencia tecnocrática, es pertinente señalar que del periodo estudiado, fue en el año 1998 cuando mayor número de microempresas fueron atendidas por los programas de la Gobernación del Estado Falcón; de un total de 1480 propuestas de negocios se seleccionaron 600 y se aprobaron 583 créditos luego de realizar talleres para elaborar proyectos, ello se explica por el hecho de que para ese año estaba planteada la campaña electoral, en la cual el Ing. José Curiel aspiró la reelección como Gobernador del Estado. Esto revela que existe una lucha entre dos modelos y que el cambio de paradigma administrativo no es un proceso mecánico.

En resumen, según información obtenida de FONDAPEMI, con el Programa de Industria Familiar se han otorgado
1246 créditos, con una inversión total de 233.264 .000 bolívares, monto del cual se estima, se han generado 1246 empleos.

A diferencia del programa anterior, la puesta en practica del PIF refleja un espacio a la incorporación del estilo político-administrativo propio del modelo gerencial tecnocrático; a pesar de que su origen no tuvo estrecha vinculación con el nivel central del gobierno, donde se promovió una orientación neoliberal a lo largo de la década de los '90. También en la Gobernación del Estado Falcón con la transición de gobierno en 1996 se hicieron esfuerzos por estar en correspondencia con las orientaciones políticas predominantes de reestructuración neoliberal de la economía.

\section{El Programa de Cajas Rurales: La gobernación toma distancia}

El apoyo gubernamental a microempresas rurales en el Estado Falcón se concreta en el año de 1998, cuando FONECRA ${ }^{9}$ promueve en calidad de coordinador, conjuntamente con el Fondo de Crédito Agrícola, la Fundación CIARA, la Coordinación de Desarrollo Económico del Estado Falcón, la Secretaría de Desa-

La referida institución desde sus inicios manejó créditos por montos estandarizados y con las exigencias tradicionales de garantia para conceder financiamiento, de hecho no implementó el Programa de Industria Familiar, como previó el Decreto de su creación; lo que demuestra su limitado apoyo a estrategias microempresariales rurales. Al respecto, uno de los funcionarios encargado de la promoción de Cajas Rurales en el Estado Falcón señala lo siguiente:"A lo largo de su trayectona, FONECRA, a pesar de ser uno de los organismos estadales financieros del país con mayores reconocimientos a nivel nacional, no contaba con una estrategia para la atención de los pequeños productores... nuestros créditos eran destinados en su totalidad para la atención de aquéllos que pudieran respaldar de manera tradicional sus créditos con hipotecas o prendas, los cuales pudieran ser catalogados de medianos y grandes agricultores" (López; 2000). 
rrollo Agrícola y FONDAPEM, el programa de Cajas Rurales.

Como antecedentes de este programa se encuentra el apoyo del Fondo de Crédito Agropecuario, la Fundación CIARA, y el Fondo Internacional para el Desarrollo Agrícola, al primer programa piloto de Cajas Rurales en Venezuela, al sur del Estado Anzoátegui, con el objetivo central de adaptar la metodología de los bancos comunales de Costa Rica ${ }^{10}$ a la realidad venezoiana. También se utilizó la experiencia recogida en las comunidades pesqueras de la Isla de Coche en el Estado Nueva Esparta, en el marco de un programa del Fondo de Crédito Agropecuarto y del programa de pesca de la Unión Europea.

La estrategia financiera del Programa de Cajas Rurales es los Bancos Comunales o Bancomunales, que de acuerdo con Raydán (1996:12), son organismos financieros locales especializados, con fines de lucro, propiedad de los usuarios y encargados de ofrecer servicios financieros a sus asociados. El capital de estas organizaciones lo componen los recursos de sus dueños hechos como aporte de capital, los ahorros que logran captar de la comunidad, los prestamos recibidos por instituciones externas y las ganancias de sus operaciones de intermediación.
Esta estrategia de financiamiento para microempresas, es adaptada $y$ readjetivada en Venezuela como "Cajas Rurales" por el Fondo de Crédito Agropecuario. Según el Manual de Formación de Promotores de Cajas Rurales (1998:4) "en la metodología de las Cajas Rurales existe un profundo respeto por el conocimiento y los valores locales, hasta el punto que la capacitación está diseñada para que sean los propios miembros de la comunidad, quienes elaboren los reglamentos que rigen todas las operaciones de las mismas".

También en este mismo documento se señala que a falta de marco jurídico especifico, las cajas rurales se registran como "Sociedades Civiles". Mucha gente tiende a confundir las cajas rurales con cooperativas de ahorro y crédito. La realidad es que en esta metodologia se utilizan principios cooperativistas, pero las cooperativas se rigen por la Ley de Cooperativas, y están bajo la supervisión de la Superintendencias de Cooperativas, mientras que las Cajas son sociedades civiles.

La mencionada estrategia financiera se caracteriza por: 1) Las decisiones sobre las condiciones financieras finales son de los socios, 2) La selección, la supervisión y la recuperación del crédito, la hacen los miembros de las Cajas,

10 Esta estrategia financiera fue desarroilada por la "Fundación Integral Campesina" (FINCA) de Costa Rica, cuyo éxito después de más de 12 años de trabajo comienza a tener fama internacional. La mencionada ONG opera como una organización civil sin fines de lucro, encargada de asisti financieramente a las organizaciones locales de senvicios de ahorro y crédito, denominados "Bancomunales". FINCA promociona, desarrolla, capacita y supervisa las operaciones financieras de los Bancomunales. Esta organización también se encarga de captar recursos en los mercados financieros, nacionales e internacionales, para transferirlos en forma de préstamos a las organizaciones locales o Bancomunales. 
basados en la capacitación recibida y en el conocimiento que los miembros tienen de las condiciones financieras, administrativas, patrimoniales y morales de los usuarios del crédito, 3) Los créditos iniciales son a corto plazo y por montos pequeños que van aumentando en la medida en que se desarrolla la experiencia con el cliente, 4) Impulsan la diversificación productiva como mecanismo para estimular el crecimiento económico y reducir los riesgos de la cartera, 5) Fomentan la capacidad de ahorro mediante el uso de mecanismos de capacitación adecuados, para vincularlos al crédito, 6) Valorizan realmente la tasa de interés para impedir distorsiones y facilitar el acceso a los sistemas financieros formales, 7) $\mathrm{Se}$ reconoce a los pequeños productores rurales, no como unidades de una sola producción, sino como unidades familiares integrales con ingreso y egresos diversos (FONECRA, 2000).

Para el funcionamiento de las Cajas Rurales promovidas por FONECRA, los socios se organizan y cumplen un proceso de capacitación orientado a crear los reglamentos de la organización ${ }^{11}$ y a manejar de manera eficiente las operaciones centrales de toda institución financiera. Una vez creados los reglamentos, hechos los aporte de capital y capacitados los socios, las Cajas comienzan las actividades financieras, concediendo créditos y movilizando ahorros entre sus asociados, en lo que se denomina Cuenta Interna. Cumplida la primera etapa de operación de manera satisfactoria las Cajas creadas pueden acceder a recursos en calidad de préstamos de distintos organismos tales como FONECRA, FONDAPEMI, Fondo de Crédito Agrícola (FCA), Instituto de Crédito Agrícola y $\mathrm{Pe}$ cuario (ICAP), etc., a través de lo que se denomina Cuenta Externa.

Normalmente las Cajas están conformadas por una asamblea de socios en donde recae la autoridad de la organización. Esta asamblea nombra una Junta Directiva que se tiene bajo su responsabilidad del quehacer diario de la organización y un comité de crédito encargado de tomar las decisiones referentes a las condiciones de financieras que se establecen para otorgar los créditos.

El capital de las Cajas está compuesto por los aportes hechos por sus socios, los ahorros movilizados por la organización, los prestamos de organizaciones externas y las ganancias de la operaciones financieras.

En el Programa de Cajas Rurales, del Estado Falcón se encuentran funcionando las Cajas Rurales: "BANCRUCES", ubicada en la población de Cabure, Municipio Petit; "SAN LUIS", ubicada en Municipio Bolivar y "PUEBLO NUEVO DE LA SIERRA". Además, actualmente está en proceso de formación la caja "MARUCUSA", en el Municipio Petit. Todas ellas promocionadas por FONECRA.

Pero también en el Estado están operando las Cajas Rurales de SAN CRISTOBAL" $y$ "EL MAPORAL" en el MU-

11 En estos reglamentos se establecen los derechos de propiedad, los mecanismos de permanencia, la estructura organizacional y funcional, la distribución de ganancias y pérdidas, los procesos para resolver conflictos y las condiciones financieras que rigen la organización. 
nicipio Federación, las cuales fueron promovidas por el Proyecto de Apoyo a Pequeños Productores y Pescadores Artesanales de la Zona Arida de los Estados Lara y Falcón (PROSALAFA).

Como puede observarse, el apoyo de la Gobernación a los microempresarios rurales con la estrategia de Cajas Rurales es exclusivamente de promotor de empresas típicamente capitalistas entre los sectores de escasos recursos, con ello el Estado, se distancia de intervenir financiando directamente, tal como lo recomiendan las tendencias neoliberales, aprovechando experiencias que han sido ensayadas a nivel internacional.

En ese orden de ideas, con este programa se plantearía tal como señala Morán (1998: 93) la subordinación de las relaciones sociales a la economía y a la apropiación exclusiva de los aumentos de la productividad por parte del capital, todo lo cual para el referido autor "...son la causa de la precariedad y el aumento de la diferencia en el seno de la sociedad...Cualquier alternativa que obvie el problema político que se esconde tras estos procesos esta condenado a quedarse en el campo de las buenas intenciones...".

\section{Reflexiones finales}

Las microempresas forman parte desde inicios de los noventa, aunque de modo interrumpido, de la agenda social de la gobernación del Estado Falcón, como una estrategia productiva, en el marco de la reestructuración económica de apertura y desregulación, para dar respuesta a los crecientes problemas sociales, tales como el desempleo y la informalidad, intensificados con las medidas de ajuste y los cuales requieren atención en aras de la gobernabilidad.

Las caracteristicas que esta política ha asumido son a nuestro juicio las siguientes; 1) Formalmente los programas buscan constituir una política social productiva que contemplaba en sus inicios estrategias de comercialización y hasta de exportación, pero en la práctica sólo un programa se plantea en la asistencia técnica, medidas para la comercialización, 2) Una clara orientación de la política en los tres programas hacia el nuevo rol que la reestructuración neoliberal le asigna al Estado, de intervención limita$\mathrm{da}$, con criterios de eficiencia, de superación de las prácticas populistas. Esta orientación ha sido favorecida por influencias y experiencias internacionales, especialmente del PNUD, que se incorporaron indirectamente a través de asesorías de organismos nacionales, 3) Este proceso ha tenido lugar con las limitaciones que le imponen las aspiraciones de los gobernadores a mantenimiento y ascenso en el poder, lo que ha conducido a mantener sobretodo en procesos electorales, prácticas populistas que ha debilitado la puesta en práctica de modo riguroso del modelo gerencial tecnocrático, propio del Estado neoliberal, 4) No se vislumbraron alternativas a favor del desarrollo económico y social integral y de profundización de la democracia como ha pretendido trasmitir el discurso de la descentralización; los grupos beneficiarios de los programas tienen una acción muy restringida si consideramos el predominio de actores tales como partidos políticos tradicionales, organismos financieros internacionales y agencias burocráticas centrales y 5) Se evidencia lo poco facti- 
ble que resultó en la década de los noventa la repolitización de los espacios públicos subnacionales, dominado por el escenario planteado como de "declive", donde la estrategia de la competitividad se ha traducido en una precarización de las condiciones de vida y de trabajo, a partir de políticas macroeconómicas de aperturas de mercados y donde no se procuran relaciones de cooperación consensadas. La situación parece tomar nuevos rumbos a partir de la importancia que en la nueva constitución se asigna a las cooperativas en la descentralización (Constitución de la República Bolivariana de Venezuela, 1999, artículo 184).

\section{Bibliografía citada}

Asamblea Nacional Constituyente (1999). Constitución de la República Bolivariana de Venezuela. Gaceta Oficial No 36.860. 30 de Diciembre. Editada por Discolar. CARACAS.

Avila, M. Tereza y Freitez, Nelson (1992). Proceso de Descentralización de los Programas de Apoyo a Microempresas: Significado, Logros, Limitaciones y Perspectivas. Ponencia presentada en las VII Jornadas de Trabajo Bloque de Gobernadores de la Región Centro Occidental, San Felipe 27-28 de Marzo de 1992.

Barrantes, César (1996). El Apoyo a la Microempresa Popular en Venezuela. ¿Hacia una Politica Social Orgánica del Estado Venezolano? Cuadernos de Investigación № 14, Escuela de Gerencia Social, Caracas.

Barrantes, César (1997). El Apoyo a la Economia Popular en Venezuela. ¿Hacia un Política Social Orgánica del Estado? Fondo Editorial Tropycos, FACES/UCV. Caracas.

Barrantes, César (1999). Organizaciones de Desarrollo Social y Estado en la Economia Popular en Venezuela. Revista Espacio Abierto Vol 8, No. 1. Maracaibo. Venezuela.

Bossier, Sergio (1989). La Descentralización: Un tema Difuso y Confuso. Documento Santiago de Chile.

Crasto, Celeste (1999). Políticas Económicas del Estado Falcón. Documento presentado en las Jomadas sobre Políticas de Promoción Económica e Industrial "Propuestas para Alcanzar un Desarrollo Productivo. 5 y 6 de Agosto de 1999. Santa Ana de Coro". Estado Falcón. Venezuela.

COPLAN (1990). EI Reto Falcón. I Plan Estratégico para el Desarrolio del Estado Falcón. Venezuela.

COPLAN (1991). El Reto Falcón. Diagnóstico del Sector Artesanal, Pequeña Mediana Industria. Coro. Estado Falcón. Venezuela.

COPLAN (1996). Un Gran Acuerdo Regional, Falcón Primero. III Plan de Desarrollo para el Estado Falcón. Venezuela.

Curiel, José (1997). Decreto Ejecutivo №. 13 de Creación del Programa de Industria Familiar. 30 de enero. Coro. Estado Falcón. Venezuela.

De Mattos, Carlos (1989). Falsas Expectativas ante la Descentralización. Localistas y Neoliberales en Contradicción. Revista Nueva Sociedad № 104. Caracas.

FONDAPEMI (1998). Memorias y Cuenta de FONDAPEMI. Coro, Estado Faicón, Venezuela.

FONDAPEMI (1999). Memorias y Cuenta de FONDAPEMI. Coro, Estado Falcón, Venezuela. 
FONDAPEMI (2000). Memorias y Cuenta de FONDAPEMI. Coro, Estado Falcón, Venezuela.

FONDAPEMI (s/f). Tríptico sobre el Programa de Industria Familia. Coro, Estado Falcón, Venezuela.

FONECRA (2000). Memorias y Cuentas de FONECRA. Coro, Estado Falcón, Venezuela.

Freitez, Nelson (1991). La Cooperación Estado-Sociedad Civil en Programas de Estímulo a la Economia Popular, Caso del Programa PAMEL. Jornadas Hispano-Venezolanas de Economía Popular. 12-14 de Noviembre. Barquisimeto, Estado Lara, Venezuela.

Iranzo, Mauricio et al (1990). Líneas Estratégicas del Programa de Promoción y Apoyo a la Economia Popular, Caso Venezuela. I Seminario Internacional de Economía Popular, $26-29$ de Noviembre de 1990, Caracas.

Mckean, Cressida (1988). Empresas Pequeñas y Microempresa: Su Eficacia e Implicaciones para la Mujer. En: La Mujer en el Sector Informal. Trabajo Femenino y Microempresas en América Latina. Compilado por Marguerite Berger y Mayra Buvinic. P.P. 147-169. Nueva Sociedad. ILDIS-Quito.

Meny Yves y Thoening, Jean (1991). Políticas Públicas y Teoría del Estado. En Revista: Documentación Administrativa. No. 224-225. Barcelona. España.

Mestre, Pedro et al (1991). Programa de Apoyo a la Microempresa del Estado Falcón (MICROEMFAL). Programa guía elaborado por el Equipo Operativo Técnico, Coro, Estado Falcón, Venezuela.

MICROEMFAL (1993). Situación de la Ejecución del Programa MICROEMFAL. Informe Técnico solicitado por COPLAN. Coro, Estado Falcón, Venezuela.

Morán, Agustín (1998). El Futuro del Trabajo, el Empleo y el Sector Voluntario. En
¿Trabajo Voluntario o Participación? Elementos para una Sociología del Tercer Sector. Coordinador Javier Jérez. Editorial Tecnos. Madrid.

Morata, Frances (1991). Políticas Públicas y Relaciones Intergubernamentales. Revista Documentación Administrativa №. 224-225. Barcelona. España.

Ochoa, Haydée (1997). La Reforma de la Administración Pública en Venezuela: Proyecto y realidad. En Revista Gestión y Política Pública, Vol. VI, Num. 1, Primer Trimestre. Revista del CIDE. México.

Ochoa, Haydée (1999). Elementos Teóricos para el Estudio de la Modernización Administrativa de las Gobernaciones. Revista Venezolana de Ciencias Sociales, Vol. 3, No 1. Cabimas. Venezuela.

Oszlak, Oscar y O'Donnell, Guillermo (1976). Estado y Políticas Estatales en América Latina: Hacia una Estrategia de Investigación. Mimeo. Argentina.

Otero, Maria (1988). Programas de los Grupos Solidarios. En: La Mujer en el Sector Informal. Trabajo Femenino y Microempresas en América Latina. Compilado por Margarite Berger $y$ Mayra Buvinic. Nueva Sociedad. ILDIS-QUITO.

Pérez Salgado (1997). El Papel de la cooperación técnica internacional en el proceso de modernización del Estado y de la gestión pública. En Revista: Reforma y Democracia No. 8, CLAD. Caracas.

Raydán, Salomón (1996). Aspectos Conceptuales de las Cajas Rurales. Fondo de Crédito Agropecuario. Mimeo. Caracas

Sánchez López, Elba Elina (1998). Impacto Socioeconómico Generado por el Programa de Industria Familiar de FONDAPEMI, en el Municipio Miranda del Estado Falcón. Tesis de Grado, 
Programa de Ingeniería Industrial. Universidad Experimental Francisco Miranda. Coro Estado Falcón.

Saraví, Gonzalo (1998). Micro y Pequeñas Empresas en los Distritos Industriales de México: La Necesidad de un nuevo Contexto Institucional. En: Sociológica Año 13, No 37. Revista de La Universidad Autónoma Metropolitana. México.

Socorro, Norma (1995). Exportaciones Microempresariales y Liberación de Mercados. Nueva Sociedad, Fundación Escuela de Gerencia Social, Fundación Eugenio Mendoza. Caracas.

Subirats, Joan (1989). Análisis de Políticas Públicas y Eficacia de la Administración. Ministerio para las Administraciones Públicas-Instituto Naciona! de Administración Pública. Madrid.

Urbaneja, Diego Bautista (1984). El Sistema Político o como funciona la máquina de procesar decisiones. En: El Caso Venezuela. Una ilusión de armonia. Ediciones IESA. Caracas.

Vilas, Carlos (2000) ¿Más allá del "Consenso de Washington"? Un enfoque desde la política de algunas propuestas del Banco Mundial sobre Reforma Institu- cional. En Revista: Reforma y Democracia. CLAD. Caracas.

YUNUS, Muhammad (1998), Hacia un Mundo sin Pobreza. Editorial Andrés Bello. Traducido por Pabio Azocar. Santiago de Chile.

Manual de Apoyo para Formación de Promotores de Cajas Rurales. Abril de 1998. Caracas.

\section{Entrevistas}

Crasto, Celeste (2000). "Reformulación de la Política de la Gobernación del Estado Falcón para el Apoyo a las Microempresas". Entrevista personal. Asesora de Desarrollo Económico. 6 de abril. Coro, Estado Falcón, Venezuela.

López, Pedro (2000) "Programa de Cajas Rurales". Entrevista personal. Promotor del Programa de Cajas Rurales, sede de FONECRA. 15 de marzo. Coro, Estado Falcón, Venezuela.

Sánchez, Rodolfo (2000). "Programa de Industria Familiar". Entrevista personal. Asistente Técnico de FONDAPEMI. 10 de marzo. Coro, Estado Falcón, venezuela. 